\title{
An Atypical Case of an Unresectable Hemangioma in the Posterior Mediastinum
}

\section{Aarab Jihan*, Lahlali Fatima Zahra, Allam Asma, Moukasse Yasmina, Sfaoua Hasna, El- Majjaoui Sanae, El-Kacemi Hanan, Tayeb Kebdani and Noureddine Benjaafar}

Department of Radiation Oncology, National Institute of Oncology, Mohammed V University, Rabat, Morocco

Corresponding author: Jihan A

$\equiv$ aarabjihan@gmail.com

Department of Radiation Oncology, National Institute of Oncology, Mohammed V University, Rabat, Morocco.

Tel: 00212662436121

Citation: Jihan A, Zahra LF, Asma A, Yasmina M, Hasna S, et al. An Atypical Case of an Unresectable Hemangioma in the Posterior Mediastinum. Insights Chest Dis. 2017, 2:1.

Received: May 03, 2018; Accepted: May 14, 2018; Published: May 18, 2018

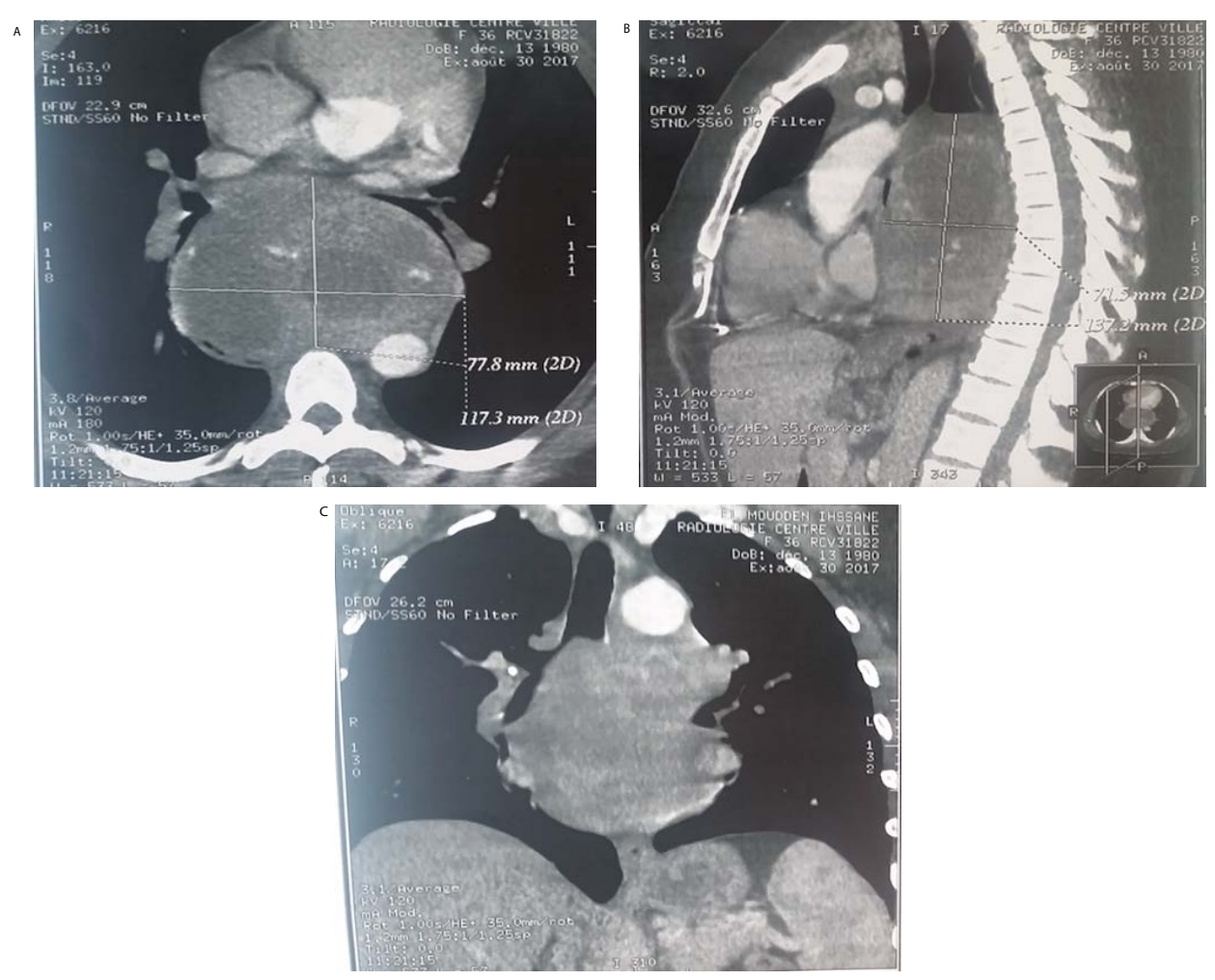

Figure 1 A) Axial plane, B) Coronal reconstruction and C) Sagittal reconstruction. 


\section{Clinical Image}

A 37-year-old woman presented to the pneumology department with 10 months history of dyspnea. There was no other local or systemic symptom. The patient medical history was unremarkable. Physical examination was normal. A chest radiograph showed a mass in mediastinum. Computed tomography (CT) of the chest revealed a heterogenous mass, well-circumscribed in the posterior mediastinum, measuring $12.7 \times 8 \times 15 \mathrm{~cm}$. In front this mass is in contact with the cardiac cavities, pushing back the left atrium, at the top it is in contact with the carina with important effect of mass on the stumps bronchial. The pulmonary arteries are permeable (Figure $\mathbf{1}(\mathbf{A}-\mathbf{C})$ ). A percutaneous fine needle aspiration biopsy of the lesion was performed under CT guidance, but it was not concluante. She was referred to thoracic surgeon for surgical intervention. A posterolateral thoracotomy was performed under general anesthesia, which revealed anenorm mass in the posterior mediastinum adhered firmly to the heart tissue, but there was no definite direct invasion, surgical excision was not possible. Histopathological examination revelated hemangioma. Radiation therapy was proposed to our patient, but she refused this therapy after the recognition of possible late risks associated with irradiation with Figure $1 \mathrm{~A}-\mathrm{C}$ showing CT appearance of hemangioma. 